\title{
Correction to: Parents' Translations of Child Gesture Facilitate Word Learning in Children with Autism, Down Syndrome and Typical Development
}

\author{
Nevena Dimitrova ${ }^{1} \cdot$ Şeyda Özçalışkan ${ }^{1} \cdot$ Lauren B. Adamson $^{1}$
}

Published online: 14 October 2017

(C) Springer Science+Business Media, LLC 2017

Correction to: J Autism Dev Disord (2016) 46:221-231

DOI 10.1007/s10803-015-2566-7

The original version of the article unfortunately contained mistake in the "Methods/Participants" section (p. 224) as " $\left[M_{\mathrm{TD}}=168.26(\mathrm{SD}=125.18)\right.$ vs. $M_{\mathrm{AU}}=172.91$ $(\mathrm{SD}=195.88)$ vs. $M_{\mathrm{DS}}=145.43$ ( $\left.\mathrm{SD}=88.78\right)$; Kruskal-Wallis, $\left.\chi^{2}(2)=2.42, p=.30\right]$, and for word types $\left[M_{\mathrm{TD}}=28.43\right.$ $(\mathrm{SD}=26.89)$ vs. $M_{\mathrm{AU}}=39.65(\mathrm{SD}=49.07)$ vs. $M_{\mathrm{DS}}=18.35$ $\left.(\mathrm{SD}=22.78) ; \chi^{2}(2)=3.15, p=.21\right]^{\prime}$.
The correct text is given below:

" $\left[M_{\mathrm{TD}}=51.91 \quad(\mathrm{SD}=59.68) \quad\right.$ vs. $\quad M_{\mathrm{AU}}=74.43$ $(\mathrm{SD}=116.01)$ vs. $M_{\mathrm{DS}}=25.26(\mathrm{SD}=39.39)$; Kruskal-Wallis, $\left.\chi^{2}(2)=3.39, p=.18\right]$, and for word types $\left[M_{\mathrm{TD}}=18.48\right.$ $(\mathrm{SD}=20.51)$ vs. $M_{\mathrm{AU}}=24.74(\mathrm{SD}=32.98)$ vs. $M_{\mathrm{DS}}=11.22$ $\left.(\mathrm{SD}=18.87) ; \chi^{2}(2)=3.58, p=.17\right]^{\prime} .$.

The online version of the original article can be found under doi:10.1007/s10803-015-2566-7.

Nevena Dimitrova

ndimitrova@gsu.edu

1 Department of Psychology, Georgia State University, P.O. Box 5010, Atlanta, GA 30303-3083, USA 